\title{
Socioeconomic status and substance use among Swiss young men: a population- based cross-sectional study
}

Eleni Charitonidi ${ }^{1}{ }^{2 *} \mathbb{D}$, Joseph Studer ${ }^{2}$, Jacques Gaume ${ }^{2}$, Gerhard Gmel ${ }^{2}$, Jean-Bernard Daeppen ${ }^{2}$ and Nicolas Bertholet ${ }^{2}$

\begin{abstract}
Background: Socioeconomic status (SES) is often inversely related to health outcomes and is likely to play a role in the use of psychoactive substances among young individuals, although little consensus exists on the association between SES and substance use.

The purpose of the study was to determine the association of three SES indicators (perceived family income, education level of participants, and parental education level) with past year use of alcohol, tobacco, cannabis, other illicit drugs and non-medical use of prescription drugs (NMPD) among Swiss young men.

Methods: Population-based cross-sectional study of 5,702 men at mean age twenty. Associations between SES indicators and substance use were assessed with regression models adjusted for age and linguistic region.

Results: Participants with average or below average perceived family income were less likely to report any use of alcohol $(O R=0.75)$ but more likely to use tobacco daily $(O R=1.31)$ and cannabis weekly $(O R=1.27)$ compared to those with perceived above average family income.

Participants whose parents had only achieved obligatory education were less likely to engage in any use of alcohol $(\mathrm{OR}=0.30)$, monthly risky single occasion drinking ( $R S O D$, defined as 6 or more drinks per occasion) $(\mathrm{OR}=0.48)$, any use of cannabis $(\mathrm{OR}=0.53)$ and other illicit drugs $(\mathrm{OR}=0.58)$, whereas those whose parents had only achieved secondary education were less at risk of engaging in cannabis $(O R=0.66$ for any use and $O R=0.77$ for more than once a week use) and other illicit drugs $(\mathrm{OR}=0.74)$ use, compared to those whose parents had achieved tertiary education. Compared to participants who completed secondary or tertiary education, those who completed only obligatory education reported a higher risk of tobacco ( $O R=1.18$ for any use, $O R=1.31$ for daily use), cannabis $(\mathrm{OR}=1.23$ for any use, $\mathrm{OR}=1.37$ for more than once a week use), and other illicit drugs $(\mathrm{OR}=1.48)$ use. No association was found between NMPD and the studied SES variables.
\end{abstract}

Conclusion: The relationship between SES and substance use was complex in this sample. Higher socioeconomic status was associated with more alcohol and other illicit drugs use, while lower socioeconomic status was related to more tobacco use. Education level and perceived family income may have different impacts on substance use and may vary by substance.

Keywords: Socio-economic status, Substance use, Alcohol, Tobacco, Illicit drugs

\footnotetext{
* Correspondence: Eleni.Charitonidi@unil.ch

'Doctoral School, Faculty of Biology and Medicine, University of Lausanne, Lausanne, Switzerland

${ }^{2}$ Alcohol Treatment Center, Department of Community Medicine and Health, Lausanne University Hospital, Beaumont 21b, P2, 02, 1011 Lausanne,

Switzerland
} 


\section{Background}

The impact of socioeconomic status (SES) on health behaviors and other outcomes has been the centerpiece of several studies [1-9]. It has been shown that death rates are higher in lower SES groups in all European countries [10-12]. Furthermore, there is growing interest in the association of SES with health-related behaviors in early adulthood, but there is conflicting evidence regarding the association of SES with substance use in this age group. The social gradient appears to differ when different indicators of SES are examined. For example, some studies have shown that some indicators of higher SES, such as parental education or family income, are associated with more use of substances [13-15], while own education level is associated with less use of substances [14]. The social gradient for a particular SES indicator may also differ across substances, since some studies have found that high SES indicators are associated with more alcohol consumption, but with less use of other substances such as tobacco [15]. Alternatively, other researchers report no significant association of different SES indicators with substance use [16]. To our best knowledge, there is no available data relating SES indicators to the non-medical use of prescription drugs.

Thus, current evidence linking SES to substance use has been mixed. Associations can differ according to the various SES indicators, such as own or parental education level, family income or perceived social position, as well as by substance used. Studying substance use across socio-economic strata will assist policy makers and public health agencies in refining preventive interventions that target substance use and abuse. The identification of inequalities in the distribution of substance use across socio-economic strata will add to the body of evidence suggesting a social gradient in health. It will inform on which population subgroups are more likely to use which substance, and indicate who should be targeted with preventive interventions. It is important to assess whether the same associations between substance use and SES indicators can be found for all substances. If one substance is not following a social gradient, this will call for preventive strategies targeting socio-economic subgroups differently for each substance. The research herein presents in detail the associations of three SES indicators (perceived family income, own education and parental education) with substance use in a sample of young men from the Swiss general population.

The first aim was to determine whether SES indicators among young adults are associated with past year prevalence of alcohol, tobacco, cannabis, and other illicit drug use, as well as non-medical use of prescription drugs (NMPD). The second aim was to examine the associations of SES indicators and intensity of substance consumption, i.e., monthly risky single occasion drinking (monthly RSOD, defined as drinking 6 or more drinks per occasion at least once a month), daily use of tobacco and more than weekly use of cannabis.

\section{Methods}

\section{Study population and setting}

The current cross-sectional study was conducted in Switzerland as part of the Cohort Study on Substance Use Risk Factors (C-SURF) [17, 18]. The participants in this large study are young Swiss men who were approached and recruited during the mandatory two-day assessment procedure to determine eligibility for military service. Because it is mandatory, virtually all males at age 19 must attend one of the recruitment centers, thus providing a unique opportunity to access a large representative sample of the general population of young men in Switzerland. Recruitment for C-SURF took place at three of the six army centers, one in the French-speaking sector (Lausanne) and two in the German-speaking sectors (Windisch and Mels). These three centers are in charge of assessing the eligibility for Swiss military service in 21 of the 26 cantons of Switzerland.

Although women are allowed to voluntarily join the military service, most do not, thus they would not represent the general population and were excluded from CSURF. Because substance use may influence eligibility in the army, there is some risk of under- or over-reporting substance abuse among attendees of the recruitment centers. Therefore all potential research participants were assured that any and all information gathered as part of the study was to be kept confidential from the army, and could not affect conscription procedures. Strong efforts were made to ensure that participants understood that the research was entirely independent of the army, which in no way could gain access to data connected to any individual.

All conscripts attending the centers were eligible for participation in C-SURF, provided they furnished written informed consent. Research staff informed potential participants (conscripts) that the study was a longitudinal survey. The goal of the study, studying substance use risk factors, was made clear to potential participants. Potential participants were also informed on the study procedures and the right to withdraw from the study at any time without penalty. Participants completed informed consent at the study recruitment site (army recruitment centers). The study questionnaires were completed by the participants after they left the army recruitment centers. The study was approved by the Ethics Committee for Clinical Research at the Lausanne University Medical School (protocol number 15/07).

Recruitment for this study took place between August, 2010 and November, 2011, during which time 13,245 center attendees were offered participation. Conscripts 
have the option of attending the recruitment centers while younger than 19, explaining some variation in age ranges observed in other studies conducted by our group at the army recruitment centers [19-21]. The 7,563 conscripts who were willing to participate received research questionnaires after leaving the recruitment centers, either in paper-pencil or online form, according to their preference.

\section{Dependent variables Alcohol use}

Alcohol use in the past 12 months was assessed with drinking frequency and quantity questions: "How often do you usually drink alcohol?" (participants were offered a range of response options: $7,6,5,4,3,2,1$ day(s) a week; 2-3 times a month; once a month or less; never) and, "How many standard drinks do you drink on average on days when you drink alcohol?"(open question) [22]. A standard drink was defined as $100 \mathrm{ml}$ of wine, $250 \mathrm{ml}$ of beer, $275 \mathrm{ml}$ of a premixed drink containing spirits or $20 \mathrm{ml}$ of spirits (each $\sim 10 \mathrm{~g}$ of ethanol), which is the commonly used definition of a standard drink in Switzerland [23]. The questionnaire included visual aids with pictures of standard drinks and labels so as to identify various container sizes.

The frequency of risky single occasion drinking (RSOD), defined as 6 or more drinks per occasion, was assessed with the following question: "How many times have you had 6 or more standard drinks with alcohol, during the same occasion" (Participants were offered a range of response options: every day or nearly every day; every week; every month; less than once a month, never). The time reference was the past 12 months [24]. Participants were later grouped into two categories: presence of monthly RSOD (every day or nearly every day, every week, every month) or absence of monthly RSOD (less than once a month, never). In Switzerland, six drinks contain $\sim 60 \mathrm{~g}$ of pure alcohol and is equivalent to the commonly used US measure of $5+$ drinks of $12 \mathrm{~g}$ per drink [25]. Our definition of RSOD is equivalent to the definition of NIAAA as well as to European standards.

Four dependent variables reflecting alcohol use over the past 12 months were used: 1) Any alcohol use over the past 12 months; 2) Presence of monthly RSOD; 3) Presence of weekly risky drinking (defined as 21 standard drinks per week or more); and 4) Weekly average consumption (mean number of standard drinks per week).

The weekly risky drinking definition used in the present study approximates the World Health Organization and other European clinical guidelines of risky drinking and the US National Institute on Alcohol Abuse and Alcoholism definition [18, 21, 23].

\section{Tobacco use}

Tobacco use was measured as any cigarette use during the past 12 months through a yes or no question ("Have you smoked cigarettes in the past 12 months?"), as well as the frequency of smoking, through the question "How often have you generally smoked cigarettes in the past 12 months?"(Participants were offered a range of 6 response options, from "every day" to "once in a month or less"). Participants were later grouped into two categories: daily use, or less. Thus, the two tobacco measures used as dependent variables were any use over the past 12 months and daily tobacco use.

\section{Cannabis use}

Cannabis use was assessed with questions included in the Cannabis Use Disorders Identification Test [26]. We identified participants reporting any use over the past 12 months and differentiated those who consumed once a week or less from those who consumed more than once a week using the following questions: "Have you used any cannabis over the past 12 months?"(yes or no question) and, "How often have you used cannabis over the past 12 months?"(with 5 response options from "monthly or less" to "every day or almost every day").

\section{Other illicit drug use}

Participants were asked about their use over the past 12 months use of the following substances: ecstasy; cocaine; heroin; and magic mushrooms through the question "Have you ever taken any of these drugs in the past 12 months? If yes, how often?"(with 3 response options "never", "1 to 3 times" and " 4 times or more").

\section{Non-medical use of prescription drugs (NMPD)}

Participants were asked about their use on any day over the last 12 months of sleeping pills, tranquilizers, painkillers, stimulants, antidepressants, and beta blockers ("Now we would like to ask you about your experience with prescribed medicine in the last 12 months that you may have decided to use of your own will-that is, either without a doctor's prescription or without a doctor telling you to use them. People use the following medicine or drugs of their own will to feel more alert, to relax or calm down, to feel better, to enjoy themselves, or to get high or just to see how they would work. Have you taken such medicine of your own will?"). Participants were offered 8 response options, varying from "never" to "4 times a week or more".

\section{Independent variables}

\section{Socioeconomic status (SES) indicators}

We used three indicators of participant SES: perceived family income, own education level, and parental education level. Perceived family income was assessed because 
there is growing evidence that subjective economic power is a better predictor of health outcomes than objective economic power [27-29]. Hence, participants were asked to report their perception of their family income ("How well off is your family compared to other families in your country?"). Participants were grouped in two categories: average or below average, and above average. Participant education level was determined as the highest education level achieved by the participants at the time of assessment, characterized as having received obligatory or elementary vocational education only or having received more than that. Parental education level was assessed by asking participants to report the highest education level achieved by their parents (either father or mother) ("What is the highest level of education your parents achieved?"). Parental education level was categorized into obligatory, secondary (vocational training or school) or tertiary (university or university of applied sciences).

\section{Analyses}

Relationships between SES indicators and substance use were first assessed using chi-square tests except for number of drinks/week for which analysis of variance testing equality of means was used. Subsequently, linear and logistic regression models adjusted for age, living environment (rural, i.e. < 10000 inhabitants vs. urban, i.e. $\geq 10000$ inhabitants) and linguistic region (German-vs. French-speaking) were fit. In our sample, the age range was 17.9-27.8. In Switzerland, the legal age for purchase of beer and wine is 16 , and 18 for hard liquors. For tobacco, the legal age for purchase varies by Canton (from no restriction to 18 years old, with most Cantons having a 16 or 18 year old legal age). Cannabis is an illegal drug in Switzerland (as the other drugs studied). Substance use is known to be associated with age. In addition, age can be associated with SES, notably the highest achieved education level. Analyses were therefore adjusted for age. Analyses were also adjusted for linguistic region and living environment since these can be associated with both SES indicators and substance use patterns. Substance use outcomes were first regressed on each SES indicators separately. Then, all SES indicators were tested simultaneously in a fully adjusted model. Finally, in order to test whether the associations of each SES indicators was moderated by other SES indicators, all two-way and three-way interactions between SES indicators were entered in the fully adjusted model. Since none of the interactions reached significance, results are not reported. In the regression models, SES indicators were contrasted as follows: average or below average vs. above average for perceived family income, obligatory or elementary vocational education only vs. more than obligatory or elementary vocational education for own education level, and tertiary vs. obligatory and secondary education for parental education level.

The dependent variables were any use of alcohol (past 12 months), prevalence of monthly risky single occasion drinking (RSOD), prevalence of weekly risky drinking, number of drinks per week, prevalence of tobacco use, cannabis use, other illicit drugs use (ecstasy, cocaine, heroin and mushrooms), as well as NMPD use (sleeping pills, tranquilizers, painkillers, stimulants, antidepressants, beta blockers).

\section{Results}

Data were collected between September 2010 and March 2012. A total of 5990 participants filled in the baseline questionnaire, of which 5,702 completed the questions on SES and were included in the present study. Details concerning the differences between consenters vs. nonconsenters, and responders vs. non-responders have been reported elsewhere [17, 18]. Table 1 presents demographic characteristics of the sample and socioeconomic status, perceived family income, own highest achieved education level and highest achieved education level of parents. A little less than half (44.5\%) of the participants reported above average perceived family income, $50.1 \%$ had completed obligatory or elementary vocational education and $49.9 \%$ had higher than obligatory education. Highest achieved parental education level was mostly secondary $(50.8 \%)$ and tertiary (42.9\%). A vast majority (92.3\%) of conscripts reported having used alcohol over the past 12 months, and nearly half of these (46.2\%) reported monthly RSOD, but only a small number of participants (6.2\%) engaged in weekly risky drinking ( 21 or more drinks per week). Tobacco was used by $47.2 \%$ of the sample, including $21.0 \%$ who reported daily use; $30.6 \%$ of the participants reported any use of cannabis during the past year, and $9.5 \%$ of these used it more than once a week. During the past year, only $6.3 \%$ had consumed at least one other illicit drug and $10.6 \%$ had taken at least one NMPD. Other than cannabis, the most frequently used illicit drug was ecstasy (3.7\%), while painkillers were the most frequently used NMPD (6.8 \%).

\section{SES indicators and substance use, unadjusted analyses}

Table 2 presents the relationship of SES indicators to substance use. Compared to those with average or below average perceived family income, participants with perceived above average family income were more likely to report any use of alcohol and monthly RSOD. However, participants with average or below average perceived family income were more likely to report both more daily tobacco use and more than once a week cannabis use than did those with above average perceived family 
Table 1 Descriptive characteristics of the sample $(N=5702)$

\begin{tabular}{|c|c|c|}
\hline & N/Mean & $\% / S D$ \\
\hline Age & 19.99 & 1.23 \\
\hline \multicolumn{3}{|l|}{ Linguistic region } \\
\hline German-speaking & 2570 & 45.1 \\
\hline French-speaking & 3132 & 54.9 \\
\hline \multicolumn{3}{|l|}{ Living environment } \\
\hline Rural (<10000 inhabitants) & 3440 & 60.3 \\
\hline Urban ( $\geq 10000$ inhabitants) & 2262 & 39.7 \\
\hline \multicolumn{3}{|l|}{ Perceived family income } \\
\hline Average or below average & 3164 & 55.5 \\
\hline Above average & 2538 & 44.5 \\
\hline \multicolumn{3}{|l|}{ Highest achieved own education level } \\
\hline Obligatory or elementary vocational education & 2858 & 50.1 \\
\hline $\begin{array}{l}\text { Higher than obligatory or elementary vocational } \\
\text { education }\end{array}$ & 2844 & 49.9 \\
\hline \multicolumn{3}{|l|}{ Parents' highest achieved education level } \\
\hline Obligatory & 362 & 6.3 \\
\hline Secondary & 2894 & 50.8 \\
\hline Tertiary & 2446 & 42.9 \\
\hline \multicolumn{3}{|l|}{ Alcohol use } \\
\hline Any use (past 12 months) & 5265 & 92.3 \\
\hline RSOD monthly or more ${ }^{a}$ & 2635 & 46.2 \\
\hline Weekly risky drinking ${ }^{b}$ & 355 & 6.2 \\
\hline Number of drinks/week (mean SD) & 7.37 & 13.14 \\
\hline \multicolumn{3}{|l|}{ Tobacco use } \\
\hline Any use (past 12 months) & 2693 & 47.2 \\
\hline Daily use & 1196 & 21.0 \\
\hline \multicolumn{3}{|l|}{ Cannabis use } \\
\hline Any use (past 12 months) & 1744 & 30.6 \\
\hline More than once a week & 543 & 9.5 \\
\hline Other illicit drugs use ${ }^{c}$ & 358 & 6.3 \\
\hline \multicolumn{3}{|l|}{ Any use (past 12 months) } \\
\hline NMPD use ${ }^{d}$ & 607 & 10.6 \\
\hline Any use (12-month use) & & \\
\hline
\end{tabular}

Note: ${ }^{a}$ Risky single occasion drinking (RSOD) defined as drinking 6 or more drinks on one occasion. b'Weekly risky drinking, defined as drinking 21 drinks per week or more. 'Ecstasy or cocaine or heroin or magic mushroom. ${ }^{d}$ Sleeping pills or tranquilizer or painkiller or stimulant or antidepressant or beta-blocker

NMPD Non-medical use of prescription drugs, SD Standard deviation

income. The prevalence of other substances use did not differ significantly.

Compared to participants who completed more than obligatory or elementary vocational education, those who completed only obligatory school or elementary vocational education reported a higher prevalence of any tobacco use, daily tobacco use, cannabis use, and other illicit drug use. Alcohol and NMDP use were not significantly different.

Participants whose parents had achieved tertiary education were more likely to report any use of alcohol compared to those whose parents had achieved only obligatory and were also more likely to report monthly RSOD and larger weekly alcohol consumption. Daily tobacco use differed across parental education levels, being highest among those whose parents had completed obligatory education only. Cannabis and illicit drug use also differed according to parental education, being highest among participants whose parents had completed tertiary education.

\section{SES indicators and substance use, adjusted analyses}

Table 3 shows the results of the alcohol use outcomes analyses, adjusted for age, living environment and linguistic region. When associations of SES indicators were tested separately, participants with average or below average perceived family income were $35 \%$ less likely to report any use of alcohol and $13 \%$ less likely to engage in monthly RSOD compared to those with an above average perceived family income. Participants whose parents had completed only obligatory education were $73 \%$ less likely to report alcohol use, $54 \%$ less likely to report monthly RSOD and reported drinking significantly fewer standard drinks (1.83, on average) in a week than did those whose parents had completed tertiary education. Participants whose parents completed secondary education were $22 \%$ less likely to report alcohol use and $11 \%$ less likely to report monthly RSOD than did those whose parents had completed tertiary education.

In the fully adjusted model (when all three SES predictors were entered simultaneously), the observed associations when each SES predictor was tested separately remained significant and of similar magnitude, except for the association between family income and monthly RSOD, and between parents' secondary education and alcohol use and monthly RSOD. Average or below average perceived family income was independently associated with a lower prevalence of alcohol use. Similarly, participants whose parents had completed obligatory education only were less likely to use alcohol and to report monthly RSOD and consumed fewer drinks per week than did those whose parents had completed tertiary education. Table 4 shows the results of the regressions predicting the other substance use outcomes, using the same models listed in Table 3. When associations of SES indicators were tested separately, average or below average perceived family income was positively associated with daily use of tobacco. Obligatory or elementary vocational education was positively associated with any tobacco use, daily tobacco use, any cannabis use, cannabis use more than once a week, and other illicit 
Table 2 Substance use outcomes as a function of perceived family income, own education and parents' education

\begin{tabular}{|c|c|c|c|c|c|c|c|c|c|c|c|c|c|c|c|c|c|}
\hline & \multicolumn{5}{|c|}{ Perceived family income } & \multicolumn{5}{|c|}{ Highest achieved own education level } & \multicolumn{7}{|c|}{ Parents' highest achieved education level } \\
\hline & \multicolumn{2}{|c|}{$\begin{array}{l}\text { Average or below } \\
\text { average }(N=3164, \\
55.5 \%)\end{array}$} & \multicolumn{2}{|c|}{$\begin{array}{l}\text { Above average }(\mathrm{N} \\
=2538,44.5 \%)\end{array}$} & \multirow[b]{2}{*}{$p$} & \multicolumn{2}{|c|}{$\begin{array}{l}\text { Obligatory or } \\
\text { elementary vocational } \\
\text { education ( } N=2858 \text {, } \\
50.1 \%)\end{array}$} & \multicolumn{2}{|c|}{$\begin{array}{l}\text { Higher than obligatory } \\
\text { or elementary } \\
\text { vocational education } \\
(N=2844,49.9 \%)\end{array}$} & \multirow[b]{2}{*}{$p$} & \multicolumn{2}{|c|}{$\begin{array}{l}\text { Obligatory }(N= \\
362,6.3 \%)\end{array}$} & \multicolumn{2}{|c|}{$\begin{array}{l}\text { Secondary }(N= \\
2894,50.8 \%)\end{array}$} & \multicolumn{2}{|c|}{$\begin{array}{l}\text { Tertiary }(N= \\
2446,42.9 \%)\end{array}$} & \multirow[b]{2}{*}{$p$} \\
\hline & $\mathrm{N} /$ mean & $\% / S D$ & N/mean & $\% / S D$ & & $\mathrm{~N} /$ mean & $\% / S D$ & $\mathrm{~N} / \mathrm{mean}$ & $\% / S D$ & & $\mathrm{~N} /$ mean & $\% / S D$ & $\mathrm{~N} /$ mean & $\% / S D$ & $\mathrm{~N} /$ mean & $\% / S D$ & \\
\hline \multicolumn{18}{|l|}{ Alcohol } \\
\hline Any use (past 12 months) & 2873 & $90.8 \%$ & 2392 & $94.2 \%$ & $<.001$ & 2653 & $92.8 \%$ & 2612 & $91.8 \%$ & .162 & 285 & $78.7 \%$ & 2681 & $92.6 \%$ & 2299 & $94.0 \%$ & $<.001$ \\
\hline RSOD monthly or more ${ }^{a}$ & 1401 & $44.3 \%$ & 1234 & $48.6 \%$ & .001 & 1322 & $46.3 \%$ & 1313 & $46.2 \%$ & .946 & 104 & $28.7 \%$ & 1338 & $46.2 \%$ & 1193 & $48.8 \%$ & $<.001$ \\
\hline Weekly risky drinking ${ }^{b}$ & 203 & $6.4 \%$ & 152 & $6.0 \%$ & .507 & 185 & $6.5 \%$ & 170 & $6.0 \%$ & .439 & 16 & $4.4 \%$ & 189 & $6.5 \%$ & 150 & $6.1 \%$ & .284 \\
\hline $\begin{array}{l}\text { Number of drinks/week } \\
\text { (mean SD) }\end{array}$ & 7.30 & 14.74 & 7.46 & 10.82 & 648 & 7.46 & 13.58 & 7.29 & 12.69 & 619 & 5.54 & 25.39 & 7.43 & 12.51 & 7.58 & 11.05 & .021 \\
\hline \multicolumn{18}{|l|}{ Tobacco use } \\
\hline Any use (past 12 months) & 1519 & $48.0 \%$ & 1174 & $46.3 \%$ & .188 & 1388 & $48.6 \%$ & 1305 & $45.9 \%$ & .043 & 163 & $45.0 \%$ & 1345 & $46.5 \%$ & 1185 & $48.4 \%$ & .244 \\
\hline Daily use & 737 & $23.3 \%$ & 459 & $18.1 \%$ & $<.001$ & 633 & $22.1 \%$ & 563 & $19.8 \%$ & .029 & 85 & $23.5 \%$ & 641 & $22.1 \%$ & 470 & $19.2 \%$ & .015 \\
\hline \multicolumn{18}{|l|}{ Cannabis use } \\
\hline Any use (past 12 months) & 945 & $29.9 \%$ & 799 & $31.5 \%$ & .189 & 912 & $31.9 \%$ & 832 & $29.3 \%$ & .030 & 87 & $24.0 \%$ & 781 & $27.0 \%$ & 876 & $35.8 \%$ & $<.001$ \\
\hline More than once a week & 329 & $10.4 \%$ & 214 & $8.4 \%$ & .012 & 291 & $10.2 \%$ & 252 & $8.9 \%$ & .089 & 33 & $9.1 \%$ & 254 & $8.8 \%$ & 256 & $10.5 \%$ & .107 \\
\hline \multicolumn{18}{|l|}{ Other illicit drugs ${ }^{\complement}$ use } \\
\hline Any use (past 12 months) & 193 & $6.1 \%$ & 165 & $6.5 \%$ & .535 & 200 & $7.0 \%$ & 158 & $5.6 \%$ & .025 & 18 & $5.0 \%$ & 159 & $5.5 \%$ & 181 & $7.4 \%$ & .010 \\
\hline \multicolumn{18}{|l|}{ NMPD $^{d}$ use } \\
\hline Any use (past 12 months) & 327 & $10.3 \%$ & 280 & $11.0 \%$ & .396 & 318 & $11.1 \%$ & 289 & $10.2 \%$ & .238 & 45 & $12.4 \%$ & 289 & $10.0 \%$ & 273 & $11.2 \%$ & .200 \\
\hline
\end{tabular}

Note. ${ }^{a}$ Risky single occasion drinking defined as drinking 6 or more drinks on one occasion. ${ }^{b}$ Weekly risky drinking, defined as drinking 21 drinks per week or more. ${ }^{c}$ Ecstasy or cocaine or heroin or magic mushroom. ${ }^{\mathrm{d}}$ Sleeping pills or tranquilizer or painkiller or stimulant or antidepressant or beta-blocker

NMPD Non-medical prescription drugs, SD Standard deviation, $P$ p value for chi square test of independence, except for number of drinks/week for which analysis of variance (testing equality of means) was used 
Table 3 Regression models of socioeconomic status predicting alcohol use outcomes

\begin{tabular}{|c|c|c|c|c|}
\hline \multirow[b]{2}{*}{ Alcohol } & \multicolumn{2}{|c|}{ Single indicator model } & \multicolumn{2}{|c|}{ Fully adjusted model } \\
\hline & $\mathrm{OR}$ & $95 \% \mathrm{Cl}$ & $\mathrm{OR}$ & $95 \% \mathrm{Cl}$ \\
\hline \multicolumn{5}{|l|}{ Any use (past 12 months) } \\
\hline Average or below average perceived family income & 0.65 & $0.53,0.80$ & 0.75 & $0.60,0.94$ \\
\hline Obligatory or elementary vocational education & 0.96 & $0.78,1.18$ & 1.01 & $0.82,1.25$ \\
\hline \multicolumn{5}{|l|}{ Parents' education (ref. tertiary) } \\
\hline Obligatory & 0.27 & $0.20,0.37$ & 0.30 & $0.22,0.41$ \\
\hline Secondary & 0.78 & $0.63,0.97$ & 0.84 & $0.67,1.05$ \\
\hline \multicolumn{5}{|l|}{ RSOD monthly or more ${ }^{a}$} \\
\hline Average or below average perceived family income & 0.87 & $0.78,0.97$ & 0.93 & $0.83,1.04$ \\
\hline Obligatory or elementary vocational education & 0.90 & $0.80,1.00$ & 0.92 & $0.82,1.02$ \\
\hline \multicolumn{5}{|l|}{ Parents' education (ref. tertiary) } \\
\hline Obligatory & 0.46 & $0.36,0.59$ & 0.48 & $0.38,0.62$ \\
\hline Secondary & 0.89 & $0.80,0.99$ & 0.91 & $0.82,1.02$ \\
\hline \multicolumn{5}{|l|}{ Weekly risky drinking ${ }^{\mathrm{b}}$} \\
\hline Average or below average perceived family income & 1.09 & $0.87,1.36$ & 1.10 & $0.87,1.38$ \\
\hline Obligatory or elementary vocational education & 1.06 & $0.84,1.33$ & 1.06 & $0.84,1.33$ \\
\hline \multicolumn{5}{|l|}{ Parents' education (ref. tertiary) } \\
\hline Obligatory & 0.75 & $0.44,1.27$ & 0.72 & $0.42,1.23$ \\
\hline \multirow[t]{2}{*}{ Secondary } & 1.06 & $0.85,1.32$ & 1.03 & $0.82,1.30$ \\
\hline & $\mathrm{b}$ & $\mathrm{p}$ & $b$ & $\mathrm{p}$ \\
\hline \multicolumn{5}{|l|}{ Number of drinks/week ${ }^{c}$} \\
\hline Average or below average perceived family income & -0.07 & .838 & 0.07 & .850 \\
\hline Obligatory or elementary vocational education & -0.09 & .808 & -0.05 & .896 \\
\hline \multicolumn{5}{|l|}{ Parents' education (ref. tertiary) } \\
\hline Obligatory & -1.83 & .014 & -1.85 & .015 \\
\hline Secondary & -0.14 & .694 & -0.16 & .671 \\
\hline
\end{tabular}

Note. All regression models are adjusted for age, living environment and linguistic region. Single indicator model: Alcohol use outcomes on SES indicator tested in separate models. Fully adjusted model: Alcohol use on perceived family income, highest achieved own education, parents' highest achieved education. ${ }^{\text {a Risky }}$ single occasion drinking defined as drinking 6 or more drinks on one occasion. ${ }^{b}$ Weekly risky drinking, defined as drinking 21 drinks per week or more. 'Linear regression models, unstandardized regression coefficient are reported $O R$ Odds ratio, $\mathrm{Cl}$ Confidence interval, $P \mathrm{P}$ value

drug use. Participants whose parents had completed only obligatory education were $46 \%$ less likely to use cannabis and $40 \%$ less likely to use other illicit drugs, compared to those whose parents completed tertiary education. Similarly, those whose parents completed secondary education were $33 \%$ less likely to use cannabis and were less prone to using other illicit drugs, compared to those whose parents completed tertiary education. Nevertheless they were more prone to report daily tobacco use. There were no significant associations of SES indicators with NMPD use.

In the fully adjusted Model, the observed associations when each SES predictor was tested separately remained significant and were of similar magnitude, except for the association between parents' secondary education and daily use of tobacco. In addition, the association of average or below average perceived family income and parents' secondary education with more than once a week cannabis use reached significance.

Those who completed obligatory or elementary vocational education were more likely to report any use of tobacco. The odds of daily tobacco use was higher among participants with average or below average perceived family income and those who had completed only obligatory school or elementary vocational education.

Participants who had completed only obligatory or elementary vocational education were more likely to report any use of cannabis, whereas those whose parents had completed obligatory or secondary education were less likely to use cannabis. Odds of using cannabis more than once a week was higher for those with average or below average perceived family income and for participants who had achieved 
Table 4 Regression models of socioeconomic status predicting tobacco, cannabis, other illicit drugs' and NMPD' use outcomes

\begin{tabular}{|c|c|c|c|c|}
\hline \multirow[b]{2}{*}{ Tobacco use } & \multicolumn{2}{|c|}{$\underline{\text { Single indicator model }}$} & \multicolumn{2}{|c|}{ Fully adjusted model } \\
\hline & $\mathrm{OR}$ & $95 \% \mathrm{Cl}$ & $\mathrm{OR}$ & $95 \% \mathrm{Cl}$ \\
\hline \multicolumn{5}{|l|}{ Any use (past 12 months) } \\
\hline Average or below average family income & 1.07 & $0.96,1.19$ & 1.10 & $0.99,1.23$ \\
\hline Obligatory or elementary vocational education & 1.17 & $1.05,1.31$ & 1.18 & $1.05,1.31$ \\
\hline \multicolumn{5}{|l|}{ Parents' education(ref. tertiary) } \\
\hline Obligatory & 0.84 & $0.67,1.05$ & 0.80 & $0.64,1.00$ \\
\hline Secondary & 0.92 & $0.83,1.03$ & 0.90 & $0.80,1.00$ \\
\hline \multicolumn{5}{|l|}{ Daily use } \\
\hline Average or below average perceived family income & 1.35 & $1.18,1.55$ & 1.31 & $1.14,1.50$ \\
\hline Obligatory or elementary vocational education & 1.33 & $1.16,1.53$ & 1.31 & $1.14,1.51$ \\
\hline \multicolumn{5}{|l|}{ Parents' education(ref. tertiary) } \\
\hline Obligatory & 1.16 & $0.89,1.52$ & 1.03 & $0.78,1.35$ \\
\hline Secondary & 1.20 & $1.05,1.37$ & 1.11 & $0.97,1.28$ \\
\hline \multicolumn{5}{|l|}{ Cannabis use } \\
\hline \multicolumn{5}{|l|}{ Any use (past 12 months) } \\
\hline Average or below average perceived family income & 0.90 & $0.80,1.01$ & 1.01 & $0.90,1.14$ \\
\hline Obligatory or elementary vocational education & 1.20 & $1.06,1.35$ & 1.23 & $1.09,1.40$ \\
\hline \multicolumn{5}{|l|}{ Parents' education(ref. tertiary) } \\
\hline Obligatory & 0.54 & $0.42,0.70$ & 0.53 & $0.41,0.68$ \\
\hline Secondary & 0.67 & $0.60,0.75$ & 0.66 & $0.59,0.75$ \\
\hline \multicolumn{5}{|l|}{ More than once a week } \\
\hline Average or below average perceived family income & 1.19 & $0.99,1.43$ & 1.27 & $1.05,1.54$ \\
\hline Obligatory or elementary vocational education & 1.36 & $1.13,1.65$ & 1.37 & $1.13,1.66$ \\
\hline \multicolumn{5}{|l|}{ Parents' education(ref. tertiary) } \\
\hline Obligatory & 0.77 & $0.52,1.13$ & 0.68 & $0.46,1.00$ \\
\hline Secondary & 0.83 & $0.69,1.00$ & 0.77 & $0.64,0.94$ \\
\hline \multicolumn{5}{|l|}{ 12-month use of other illicit drugs ${ }^{a}$} \\
\hline Average or below average perceived family income & 0.89 & $0.71,1.10$ & 0.96 & $0.77,1.21$ \\
\hline Obligatory or elementary vocational education & 1.45 & $1.15,1.83$ & 1.48 & $1.18,1.87$ \\
\hline \multicolumn{5}{|l|}{ Parents' education(ref. tertiary) } \\
\hline Obligatory & 0.60 & $0.36,0.98$ & 0.58 & $0.35,0.97$ \\
\hline Secondary & 0.74 & $0.59,0.92$ & 0.74 & $0.59,0.93$ \\
\hline \multicolumn{5}{|l|}{ 12-month use of NMPD ${ }^{b}$} \\
\hline Average or below average perceived family income & 0.94 & $0.79,1.11$ & 0.95 & $0.79,1.13$ \\
\hline Obligatory or elementary vocational education & 1.15 & $0.96,1.38$ & 1.16 & $0.96,1.39$ \\
\hline \multicolumn{5}{|l|}{ Parents' education (ref. tertiary) } \\
\hline Obligatory & 1.08 & $0.77,1.52$ & 1.09 & $0.77,1.54$ \\
\hline Secondary & 0.89 & $0.75,1.06$ & 0.90 & $0.75,1.08$ \\
\hline
\end{tabular}

Note. All regression models are adjusted for age, living environment and linguistic region. Single indicator model: Substance use on SES indicator tested in separate models. Fully adjusted model: Substance use on perceived family income, highest achieved own education, parents' highest achieved education. ${ }^{a}$ Ecstasy or cocaine or heroin or magic mushroom. ${ }^{b}$ Sleeping pills or tranquilizer or painkiller or stimulant or antidepressant or beta-blocker $O R$ Odds ratio, $C I$ Confidence interval, $P$ P value, NMPD Non-medical prescription drugs

only obligatory or elementary vocational education but was lower for those whose parents had completed secondary education.
The odds of using other illicit drugs was significantly higher for participants who had completed only obligatory or elementary vocational education but was lower 
for those whose parents had completed obligatory or secondary education. There were no significant associations of SES indicators with NMPD use.

\section{Discussion}

The first finding of this study was that higher perceived family income and parental education were associated with greater odds for alcohol use, while own education did not seem to be associated with the risk for alcohol use. Higher parental education was associated with a heavier use (monthly RSOD and weekly drinking). Lower perceived family income and own education were associated with greater odds of daily tobacco use. For cannabis, high levels of parental education and lower own education were associated with greater odds of both any use and frequent use (i.e., more than once a week), while lower perceived family income was associated with more frequent use. For other illicit drug use, higher parental education and lower own education were associated with more use, while perceived family income was not related. There was no association of non-medical use of prescription drugs with any of the three SES indicators used in this study. Most of the observed associations and the magnitude of the observed associations remained similar in the fully adjusted model, suggesting independent associations.

Our results confirm what has been shown in other studies. Humensky et al. [13], in a longitudinal study of 20,745 students (ages 12-18) who were subsequently monitored (ages 18-27), showed that individuals with a college educated parent were more likely to engage in monthly RSOD, and cannabis and cocaine use in early adulthood than did those with a high school educated parent. Similarly, individuals with higher household incomes were found to be more prone to monthly RSOD, and cocaine use. In a sample of young adults, Patrick et al. [15] also showed that alcohol and cannabis use in young adulthood were associated with higher parental education.

In a cross-sectional survey conducted in Switzerland, Abel et al. [14] found in a sample of 31,424 males (ages 18-25) that those who had finished obligatory education only were more likely to use alcohol in early adulthood compared to those with more education (OR 1.56). For parental education, the association did not follow a social gradient. Similar to our study, males whose parents had completed only obligatory education were less likely to report risky drinking $(\mathrm{OR}=0.5)$. Additionally, males from households with lower family income were less likely to report risky drinking $(\mathrm{OR}=0.81)$ than were those from wealthier households.

Other studies have also demonstrated unique patterns in the relationship of alcohol to SES indicators [14, 3033] that are different than those found for other substances. This might be explained by greater social acceptability of drinking, as well as a need for feelings of both belonging and of independence among young adults, that are enhanced by alcohol use [34].

The present study has some limitations. First, the sample consists of males only, and gives no information regarding the association of SES indicators and substance use among women. In addition, this research is based solely on assessments that were self-reported, precluding the use of any biological measures of substance use. Self-reports are potentially influenced by social desirability and recall biases. Also, potential differences between study participants and the source population may limit the generalizability of the study results. In C-SURF, as shown by Studer et al. $[17,18]$, potential differences in substance use may exist among individuals who agreed to participate in the study and those who were approached but refused to participate in the study. Nonetheless, in that large study the differences observed between consenters and non-consenters were small, even though non-consenters reported higher substance use than did consenters.

When looking at participants own education, a social gradient appears present for most substances. Nevertheless this gradient is no longer present when looking at the participant parents' education level. Independently of one's own education level, parental education is likely to play a role in which substances are used and how. This may be explained by a differing perception of alcohol, tobacco and other drugs among parents from different educational backgrounds. Depending on their education level, parents may be more permissive towards a given substance and less towards another. The perception of one's family economic power was associated with specific substance use patterns (more alcohol use for those perceiving their family has having stronger economic power, more cannabis and tobacco use for those perceiving their family as having weaker economic power). Depending on the SES strata, some substances may be considered more acceptable than others, and the perceived risks related to these substances may be influenced by the SES. Hypotheses for these differences, such as differing perceptions, norms, feelings of belonging, access and availability, etc. should be tested specifically. Studying the mechanisms behind the associations observed in the present study would help develop targeted public health interventions. Nevertheless, the observed differences inform on how to refine preventive intervention for specific population groups.

With regard to generalizability, findings should be interpreted with the Swiss context in mind. The legal age for purchase of alcohol and tobacco, and the illegal status of cannabis and other drugs may differ in other countries. Even though we used the perceived family 
income as an indicator of SES, the average income in Switzerland is high. The Swiss education system might also differ from what can be found in other countries. Nevertheless, we think our results provide important knowledge: associations between SES and substance use may differ by substance. As such, preventive intervention or public health initiatives have to target alcohol, tobacco and other drugs specifically in the different socioeconomic strata.

Notwithstanding the above limitations, the current study has some notable strengths. It was comprised of a large sample of 20-year-old males coming from diverse geographical and socioeconomic backgrounds in Switzerland. It included the assessment of several SES indicators and their associations with different licit and illicit substances, and provided indications on associations of non-medical use of prescription drugs with SES that are seldom studied.

\section{Conclusions}

Economic power (family income) appears to be associated with higher odds of using alcohol, but with lower odds of using cannabis and tobacco daily. Higher parental education level was fairly consistently associated with increased odds of using any substance except tobacco, and is possibly a reflection of varying perceptions regarding alcohol, tobacco, cannabis, and other drug use across different educational backgrounds. For the most part, the education level of participants was negatively associated with the odds of using any substance except alcohol (i.e., the lower the education level, the greater the substance use risk). Thus, the social gradient appears to differ according to the SES indicator and to the substance involved. It would be worthwhile to conduct further investigations that concentrate specifically on the perceptions of various licit and illicit substances among young individuals (as well as among their parents). The findings herein could be used to design better prevention interventions in the future. Appropriate target groups could be assembled, using the knowledge gained thus far regarding SES variables and their relationship to substance use. Notably, alcohol use does not follow the same pattern of association with lower socio-economic indicators as other substances. It should not be grouped with tobacco, cannabis and other drugs. More research is needed on norms, perceptions, acceptability of these substances across SES strata to increase the efficacy of preventive interventions and to match the intervention content to the specifics of each SES stratum. Also, a higher parental education level appear associated with the use of illicit drugs (including cannabis) and binge drinking, which questions how unhealthy substance use patterns are perceived among the more highly educated.
Our results should encourage more research on how various licit and illicit drugs are favorably (or unfavorably) perceived within the general population. Researchers and public health agencies should therefore not take for granted the current popular notion that low SES is necessarily a detrimental factor influencing the consumption of various psychotropic substances.

\section{Ethics approval}

The study was approved by the Ethics Committee for Clinical Research of the Lausanne University Medical School. C-SURF was approved by the Ethics Committee for Clinical Research of the Lausanne University Medical School (protocol number 15/07).

\section{Abbreviations}

SES: socioeconomic status; NMPD: non-medical use of prescription drugs; OR: odds ratio; Cl: confidence interval; RSOD: risky single occasion drinking; C-SURF: cohort study on substance use risk factors.

\section{Competing interests}

The authors declare that they have no competing interests

\section{Authors' contributions}

Conception and design of the present study: EC, NB, JBD, GG, JS, JG. Acquisition of data: GG, JBD, JS, NB. Design of the analyses: JS, EC, NB, GG. Analysis of data: JS. Interpretation of data: EC, JS, NB, JG, GG, JBD. Main drafting of the manuscript: EC, NB. Revision of manuscript: EC, NB, JBD, GG, JS, JG. All authors read and approved the current version of the manuscript. GG is the principal investigator of C-SURF.

\section{Acknowledgments}

The authors would like to thank Charlotte Eidenbenz, project manager for C-SURF, and the C-SURF team for their work on the study, as well as the participants for their time and dedication to the study.

\section{Funding}

C-SURF was funded by the Swiss National Science Foundation (33CSCO-122679, Pl: G Gmel).

Researchers were independent from the Swiss National Science Foundation.

Data

Data is available upon request. C-SURF is an ongoing cohort study. Researchers interested in C-SURF data (beyond the data presented in the current article) can submit research projects (www.c-surf.ch).

Received: 28 July 2015 Accepted: 9 March 2016

Published online: 14 April 2016

\section{References}

1. Chen E, Miller GE. Socioeconomic status and health: mediating and moderating factors. Annu Rev Clin Psychol. 2013;9:723-49.

2. Stringhini $\mathrm{S}$ et al. Association of socioeconomic position with health behaviors and mortality. JAMA. 2010;303(12):1159-66.

3. Mackenbach JP. The contribution of medical care to mortality decline: McKeown revisited. J Clin Epidemiol. 1996;49(11):1207-13.

4. Mackenbach JP, Stronks K, Kunst AE. The contribution of medical care to inequalities in health: differences between socio-economic groups in decline of mortality from conditions amenable to medical intervention. Soc Sci Med. 1989:29(3):369-76.

5. Adler NE, Stewart J. Preface to the biology of disadvantage: socioeconomic status and health. Ann N Y Acad Sci. 2010;1186:1-4.

6. Braveman P, Gottlieb L. The social determinants of health: it's time to consider the causes of the causes. Public Health Rep. 2014;129 Suppl 2:19-31. 
7. Camargo KRD. Closing the gap in a generation: Health equity through action on the social determinants of health. Glob Public Health. 2011;6(1):102-5.

8. Marmot M, Bell R. Fair society, healthy lives. Public Health. 2012;126 Suppl 1:S4-10

9. Marmot $\mathrm{M}$ et al. Social inequalities in health: next questions and converging evidence. Soc Sci Med. 1997;44(6):901-10.

10. Menvielle $\mathrm{G}$ et al. [Socioeconomic inequalities in mortality in Europe]. Med Sci (Paris). 2009;25(2):192-6.

11. Mackenbach JP et al. Socioeconomic inequalities in health in 22 European countries. N Engl J Med. 2008;358(23):2468-81.

12. Mackenbach JP et al. Widening socioeconomic inequalities in mortality in six Western European countries. Int J Epidemiol. 2003;32(5):830-7.

13. Humensky JL. Are adolescents with high socioeconomic status more likely to engage in alcohol and illicit drug use in early adulthood? Subst Abuse Treat Prev Policy. 2010;5:19.

14. Abel T, Hofmann K, Schori D. Social and regional variations in health status and health behaviours among Swiss young adults. Swiss Med Wkly. 2013; 143:w13901.

15. Patrick ME et al. Socioeconomic status and substance use among young adults: a comparison across constructs and drugs. J Stud Alcohol Drugs. 2012:73(5):772-82.

16. Hamilton HA, Noh S, Adlaf EM. Perceived financial status, health, and maladjustment in adolescence. Soc Sci Med. 2009;68(8):1527-34.

17. Studer $J$ et al. Need for informed consent in substance use studies-harm of bias? J Stud Alcohol Drugs. 2013;74(6):931-40.

18. Studer $J$ et al. Examining non-response bias in substance use research-are late respondents proxies for non-respondents? Drug Alcohol Depend. 2013;132(1-2):316-23.

19. Bertholet $\mathrm{N}$ et al. Perception of the amount of drinking by others in a sample of 20-year-old men: the more I think you drink, the more I drink. Alcohol Alcohol. 2011;46(1):83-7.

20. Daeppen JB et al. Efficacy of brief motivational intervention in reducing binge drinking in young men: A randomized controlled trial. Drug Alcohol Depend. 2011;113(1):69-75.

21. Gmel $\mathrm{G}$ et al. Who drinks most of the total alcohol in young men-risky single occasion drinking as normative behaviour. Alcohol Alcohol. 2008;43(6):692-7.

22. Rehm J. Measuring quantity, frequency, and volume of drinking. Alcohol Clin Exp Res. 1998;22(2 Suppl):4S-14S.

23. Adam A et al. Age of first alcohol intoxication: association with risky drinking and other substance use at the age of 20. Swiss Med Wkly. 2011;141:w13226.

24. Saunders JB et al. Development of the Alcohol Use Disorders Identification Test (AUDIT): WHO Collaborative Project on Early Detection of Persons with Harmful Alcohol Consumption-II. Addiction. 1993;88(6):791-804.

25. Gmel G, Rehm J. Harmful alcohol use. Alcohol Res Health. 2003;27(1):52-62.

26. Adamson SJ, Sellman JD. A prototype screening instrument for cannabis use disorder: the Cannabis Use Disorders Identification Test (CUDIT) in an alcohol-dependent clinical sample. Drug Alcohol Rev. 2003;22(3):309-15.

27. Singh-Manoux A, Marmot MG, Adler NE. Does subjective social status predict health and change in health status better than objective status? Psychosom Med. 2005;67(6):855-61.

28. Piko B, Fitzpatrick KM. Does class matter? SES and psychosocial health among Hungarian adolescents. Soc Sci Med. 2001;53(6):817-30.

29. Frojd $\mathrm{S}$ et al. Perceived financial difficulties and maladjustment outcomes in adolescence. Eur J Public Health. 2006:16(5):542-8.

30. Kendler KS et al. Socioeconomic status and alcohol-related behaviors in mid- to late adolescence in the avon longitudinal study of parents and children. J Stud Alcohol Drugs. 2014;75(4):541-5.

31. Finch KA et al. Subjective social status and substance use severity in a young adult sample. Psychol Addict Behav. 2013;27(3):901-8.

32. Poonawalla IB et al. Family income trajectory during childhood is associated with adolescent cigarette smoking and alcohol use. Addict Behav. 2014; 39(10):1383-8

33. Harrell ZA, Huang JL, Kepler DM. Brief report: Affluence and college alcohol problems: the relevance of parent- and child-reported indicators of socioeconomic status. J Adolesc. 2013;36(5):893-7.

34. Capraro RL. Why college men drink: alcohol, adventure, and the paradox of masculinity. J Am Coll Health. 2000;48(6):307-15.

\section{Submit your next manuscript to BioMed Central and we will help you at every step:}

- We accept pre-submission inquiries

- Our selector tool helps you to find the most relevant journal

- We provide round the clock customer support

- Convenient online submission

- Thorough peer review

- Inclusion in PubMed and all major indexing services

- Maximum visibility for your research

Submit your manuscript at www.biomedcentral.com/submit
C Biomed Central 